\title{
OTIOTOMICS
}

Revista de economía, empresa y sociedad

Dosier «Vectores de sostenibilidad: visiones desde la economía»

\section{Urbanización, ocupación del suelo e infraestructuras de soporte}

\section{Mireia Hernández Asensi}

Directora de proyectos en PCE Engenharia. Profesora colaboradora en el Máster de Gestión de la Ciudad y Urbanismo (UOC)

\section{Fduard J. Alvarez-Palau}

Profesor de los Estudios de Economia y Empresa (UOC)

RESUMEN En el año 2015, la Asamblea General de la ONU aprobó la Agenda 2030. Consensuó 17 objetivos de desarrollo sostenible (ODS), cuya finalidad era repensar las pautas de desarrollo en todo el planeta. Las ciudades, como aglomeraciones poblacionales y centros de producción, son actores directamente interpelados. Los patrones de crecimiento seguidos durante los últimos años muestran una clara explosión de su trama urbana y una ocupación del territorio sin precedentes. Tanto es así que determinados sectores urbanos degradados son abandonados en detrimento de sectores de nueva construcción ubicados en la periferia urbana. Las connotaciones de este fenómeno son múltiples, pero es importante centrar el foco en la insostenibilidad de un modelo de crecimiento urbano sustentado en la promoción urbanística, la ocupación del suelo y unas infraestructuras de transporte que no han sido planificadas de manera coordinada con estos proyectos urbanísticos. Con todo, este artículo reflexiona sobre las externalidades de este fenómeno, y plantea algunas reflexiones para ayudar a hacer la transición hacia un modelo de ciudad más sostenible.

PALABRAS CLAVE urbanización; ocupación del suelo; rehabilitación; infraestructuras; transporte; centro-periferia; sostenibilidad 


\section{Urbanization, land occupancy and supporting infrastructure}

ABSTRACT In 2015, the UN General Assembly approved the 2030 Agenda. There was an agreement on 17 Sustainable Development Goals (SDGs), the purpose of which was to reverse development patterns around the planet. Cities, as population agglomerations and production centres, are directly challenged actors. The growth patterns followed in recent years show a clear explosion of the urban fabric, and an unprecedented land occupancy. So much so that certain degraded urban sectors are being abandoned to the detriment of new construction sectors located in the urban periphery. The connotations of this phenomenon are multiple, but it is important to focus on the unsustainability of an urban growth model based on urban development, land occupancy and transport infrastructures that have not been planned in coordination with these urban projects. Based on the foregoing, this article reflects on the externalities of this phenomenon, and raises some reflections to help transition towards a more sustainable city model.

KEYWORDS urbanization, land occupation, rehabilitation, infrastructures, transport, center-periphery, sustainability

\section{Introducción}

Según datos de la ONU, en 2018, la población mundial residente en zonas urbanas llegó al 55 \% del total, en contraste con el 30 \% de 1950 (ONU, 2018). Norteamérica (82 \%), América del Sur y el Caribe (81 \%) y Europa (74 \%) lideran esta tendencia. El gran problema es que Asia (50 \%) y África (43 \%) experimentan una explosión demográfica sostenida desde finales del siglo XX (ONU, 2019). Y todo parece indicar que este crecimiento se concentrará mayoritariamente en las áreas urbanas, donde se desbordarán las estructuras de las ciudades actuales y se crearán nuevas megalópolis. La principal preocupación es que este crecimiento se lleve a cabo de manera informal o poco planificada, replicando los errores del pasado en términos de ocupación del suelo y de sostenibilidad ambiental de los nuevos desarrollos. Debemos recordar que los asentamientos urbanos perduran en el tiempo y, por lo tanto, resulta más sencillo planificarlos a priori que rehabilitarlos a posteriori. Esto nos lleva a preguntarnos si habría que tomar medidas por lo que se refiere a esta cuestión. Y no parece disparatado. La propia ONU, por medio de los ODS, contempla medidas para lograr «ciudades y comunidades sostenibles» (ODS-11), pero la falta de instrumentos para ejecutar las recomendaciones puede convertirlas en papel mojado. De hecho, una de las críticas más extendidas a la Agenda 2030 es que se hable de repensar dinámicas sin abordar una de las principales causas de la insostenibilidad del sistema: el crecimiento poblacional (Kopnina, 2015). En cualquier caso, y con los instrumentos disponibles, es obvio que tanto las migraciones como los procesos de transformación urbana deben ser abordados a corto plazo si realmente se quiere promover un cambio de modelo.

Este artículo tiene como objetivo analizar el impacto de este proceso de urbanización acelerado sobre el crecimiento espacial de las ciudades occidentales. Para hacerlo, reflexiona sobre los diferentes procesos de urbanización, valora cómo se adaptan las ciudades y sus infraestructuras de soporte, y propone medidas que permitan hacer la transición hacia un modelo de crecimiento urbano más sostenible. 


\section{Procesos catalizadores de la urbanización y la ocupación del suelo}

La aceleración del fenómeno de urbanización en el mundo supone crecimientos desmedidos en las ciudades. Desde mediados de siglo XX, hemos presenciado cómo el crecimiento poblacional ha llevado a una expansión inaudita de las tramas urbanas, tanto de forma continua como dispersa, y ha generado nuevas necesidades y externalidades que hay que afrontar. La antropización del territorio es probablemente la más evidente y problemática. Una vez que se construye, es decir, que se transforma el territorio natural como consecuencia de la actividad humana, resulta prácticamente imposible volver al estado natural previo. Este crecimiento se basa en tres procesos demográficos: 1) migraciones campo-ciudad; 2) migraciones centro-periferia, y 3) migraciones entre áreas urbanas, tanto a escala nacional como internacional.

Los procesos migratorios tradicionales de las ciudades españolas, causados por el éxodo campo-ciudad, comportaron la colmatación de la trama urbana hasta sus límites físicos, históricamente las murallas, lo que dio lugar a ciudades compactas y con una elevada densidad poblacional. Tanto es así que esta tipología de ciudad llevó a la sobreocupación del espacio construido, lo que generó problemas de hacinamiento e insalubridad. A principios del siglo XX, surgieron los movimientos utópicos internacionales de la ciudad moderna y la ciudad jardín, que intentaban dar respuesta a este problema mediante un esponjamiento urbano, una segregación de los usos del suelo y una creciente preocupación por la higiene pública. Pero, en el caso español, fueron las políticas de ensanche y de promoción de vivienda pública, especialmente en la periferia urbana, las que permitieron dar respuesta a las necesidades urbanas del momento.

A partir de la transición democrática, se optó por un cambio de modelo. Se liberalizó el suelo y se desplegó un nuevo marco normativo de planificación urbanística con competencias transferidas a las administraciones locales. En este nuevo contexto, los gestores públicos se limitaban a regular y supervisar, y otorgaban a los promotores inmobiliarios buena parte del poder de decisión sobre cuándo y cómo crecían las ciudades (Herce, 2013). El desarrollo urbanístico de nuevos sectores urbanos, normalmente fuera de la trama urbana consolidada, incentivó las migraciones centro-periferia, y provocaron la explosión territorial de la ciudad. Empezaron a surgir las primeras regiones metropolitanas y, en pocos años, se pasó a entender la ciudad como un conglomerado urbano policéntrico (Alvarez-Palau et al., 2019). Pero las migraciones centro-periferia se manifiestan de manera diferente dependiendo del territorio. Por un lado, las migraciones causadas por la falta de suelo asequible para vivienda, con formación de sectores periféricos especializados en usos residenciales y ocupados por población de renta baja, las ciudades dormitorio. En estos casos, se mantiene una dependencia total del centro debido a la falta de servicios y de oportunidades laborales. También se dan migraciones hacia la ciudad difusa, motivadas por la búsqueda de una mejor calidad de vida, con formación de ciudades satélite planificadas más alejadas del centro, con menos densidad, una buena dotación de recursos y ocupadas por población de renta media-alta. En este último caso, la periferia tiene dinámicas propias y solo depende del centro para determinadas cuestiones económicas, puesto que la ciudad continúa siendo el gran generador de puestos de trabajo cualificados. Incluso aparece una tercera tipología, las ciudades de vacaciones, con un predominio de segundas residencias que solo se utilizan esporádicamente. Estos fenómenos nos están llevando hacia un despoblamiento gradual del centro de la ciudad y una urbanización más pronunciada en la periferia.

La figura 1 permite ejemplificar las consecuencias territoriales de parte de estos procesos. El crecimiento de la ciudad de Barcelona entre 1990 y 2018 muestra la colmatación de la ciudad central y la explosión de la trama urbana hacia el área metropolitana. En el caso de la ciudad de Río de Janeiro, el crecimiento entre 1975 y 2018 muestra un comportamiento ligeramente diferente. El no relleno del término municipal ha permitido un fuerte crecimiento en el barrio de Barra de Tijuca y al noroeste de la ciudad, donde el suelo es más barato. Por otro lado, también se muestra el crecimiento de sectores alrededor de la zona sur y centro, que se corresponden con áreas de riesgo geológico o hidrológico, donde se ha producido crecimiento informal en forma de favelas.

Lo que resulta curioso es que algunas de las dinámicas recientemente observadas muestran una cierta atenuación. El tercer tipo de migraciones, normalmente de carácter internacional, pasa a tener un papel más relevante en el desarrollo de las ciudades globales, es decir, en las urbes con suficiente proyección y visibilidad. Por un lado, las ciudades líderes en innovación, captación de inversión y atracción de talento ven cómo el poder adquisitivo de los recién llegados les permite establecerse en las zonas urbanas más nobles. Y es aquí cuando la llegada de nuevo capital permite rehabilitar barrios céntricos degradados. En cambio, los inmigrantes económicos y los refugiados, ya sea por conflictos bélicos o por las consecuencias del cambio climático, tienden a instalarse en barrios marginales de renta baja y sin opción a ser rehabilitados, lo que cronifica las desigualdades urbanas. 
Figura 1. Arriba, evolución de la trama urbana en la región metropolitana de Barcelona entre 1990 y 2018. Debajo, evolución de la trama urbana del municipio de Río de Janeiro entre 1975 y 2018

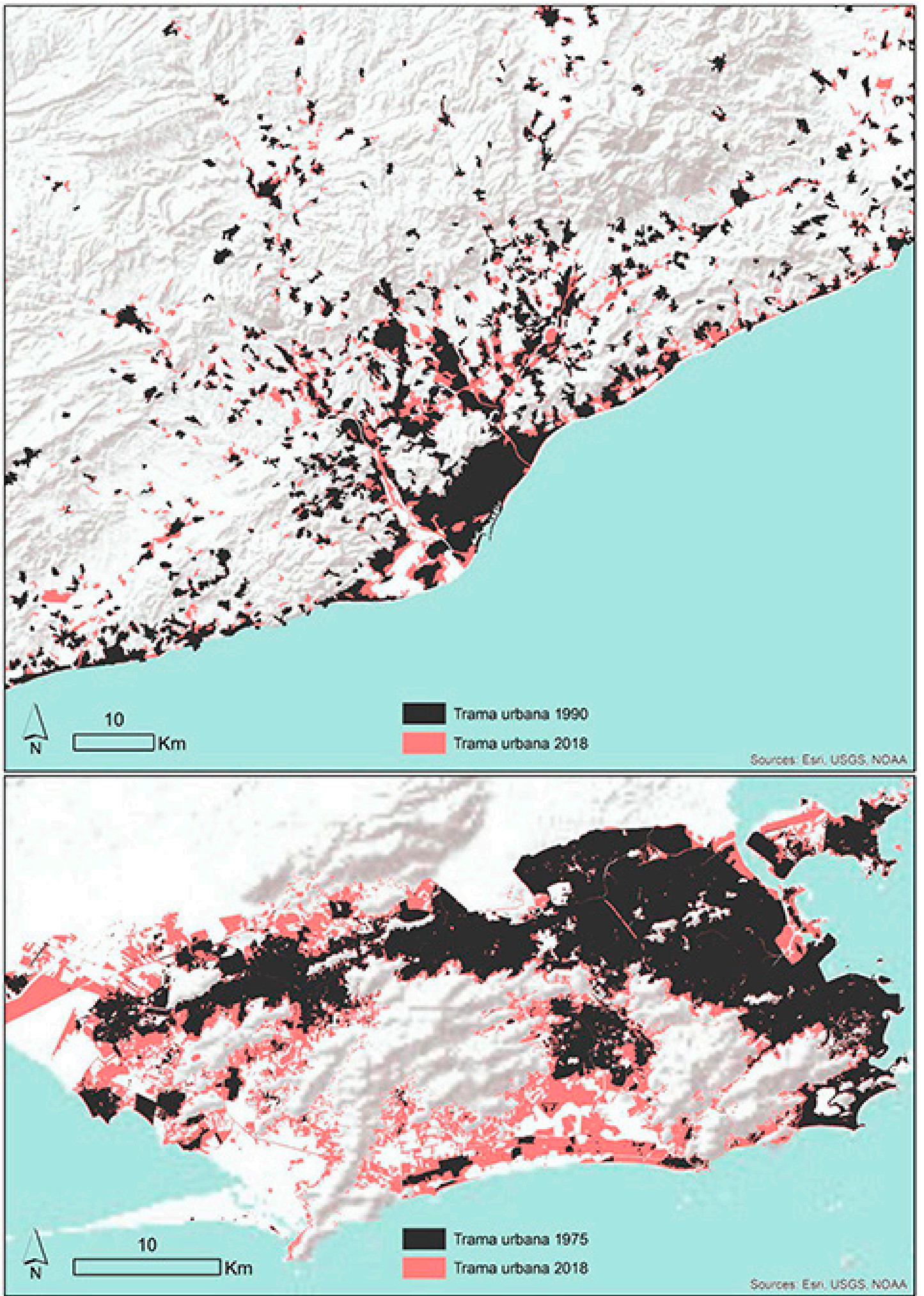

Fuente: Corine Land Cover (arriba); Instituto Municipal de Urbanismo Pereira Pasos - IPP (debajo) 


\section{Políticas de transformación y renovación del espacio urbano consolidado}

En términos económicos, las ciudades se han convertido en los principales generadores de PIB a escala mundial, incluso en los países en vías de desarrollo, pero los recursos para hacer frente a sus retos de transformación no acompañan. Las principales necesidades de la ciudad central varían dependiendo de su proyección internacional. Las ciudades de primer orden mundial están afectadas por procesos de gentrificación urbana, con alta competencia entre actividades económicas y que llevan a la expulsión de las capas de población más desfavorecidas. Las ciudades de segundo orden, en cambio, sufren la degradación urbana de determinados sectores y la inoperatividad de los instrumentos públicos de renovación urbana, lo que también acaba comportando procesos migratorios hacia la periferia.

El caso barcelonés, por ejemplo, sirve para ejemplificar la primera tipología. Con una alta proyección internacional, la ciudad ve cómo primeras marcas mundiales se disputan los escaparates en sus calles más exclusivas. También hay competencia por el suelo de oficinas entre las empresas de base tecnológica para instalar una delegación en la ciudad. En términos de suelo residencial, se observa un crecimiento sostenido en el tiempo del precio del metro cuadrado construido, especialmente de obra nueva. Esto no evita, no obstante, que no haya externalidades. Se suceden continuamente los cierres de tiendas tradicionales del centro histórico, para acabar abriendo franquicias. La proliferación de hoteles, oficinas y residencias, con más potencial de captación de valor añadido, dificulta el desarrollo de nuevo suelo residencial. Y la llegada de recién llegados con alto poder económico les permite pagar precios desmesurados por la vivienda, lo que deja los locales sin más opción que mudarse a la periferia. A todo esto, se suman fenómenos complementarios de mercantilización de la vivienda, como los pisos turísticos, que, por medio de plataformas digitales, obtienen un retorno más alto por el alquiler de corta duración que el que conseguirían en el mercado tradicional. De este modo, acaban reduciendo la oferta de vivienda para los residentes. En definitiva, los procesos de gentrificación han llevado, muchas veces, a una elitización de la ciudad central, con el aumento del precio de la vivienda y una alteración completa del perfil de los residentes. Las administraciones públicas tienen poco margen de maniobra sobre el funcionamiento del mercado y, por lo tanto, difícilmente pueden amortiguar su impacto sobre los ciudadanos.

En ciudades de segundo orden, los problemas son radicalmente diferentes. Son pocas las ciudades que no hayan sufrido un proceso de deterioro del núcleo histórico. La falta continuada de inversión, el impacto generado por infraestructuras mal integradas o, incluso, la elevada concentración de actividades de poco valor añadido generan espacios de baja calidad, normalmente asociados con población de renta baja, donde se producen procesos de degradación urbana. También se dan estos fenómenos en la periferia urbana, donde algunos barrios de vivienda popular impulsados a mediados de siglo Xx han sufrido un proceso de deterioro y marginalización. La renovación de estos sectores no siempre es fácil dadas las herramientas de planeamiento vigentes. En España, por ejemplo, se continúa trabajando con instrumentos estáticos, puesto que cualquier cambio de calificación del suelo ha de pasar por un proceso de modificación de la normativa urbanística, lo que acaba alargando el proceso y dificultando la captación de recursos financieros. Este enfoque destaca con el de otros países, como Brasil, que ha optado por instrumentos más dinámicos, y donde es común utilizar operaciones urbanas consorciadas para revitalizar los sectores urbanos estratégicos. Debido a la falta de recursos propios, los órganos públicos incentivan que sean las empresas privadas las que asuman los costes de la transformación urbana a cambio de otorgarles una edificabilidad más grande en las áreas afectadas.

\section{Infraestructuras como soporte territorial de la periferia urbana}

La expansión de la ciudad más allá de sus límites físicos ha sido posible gracias a las redes de infraestructuras, que soportan las relaciones entre centro y periferia. Las infraestructuras de transporte se pueden considerar como ejes de penetración en el territorio, elementos que rompen la fricción del movimiento, al otorgar mejores niveles de accesibilidad y conectividad. Los nuevos paradigmas teóricos dan a estas redes un papel capital en la nueva organización geográfica. Variaciones de velocidad llevan a valores del suelo diferenciados y, por lo tanto, diferentes expectativas para las actividades económicas (Herce, 2002). La proximidad física deja de ser la variable determinante. En términos geográficos, el concepto muerte de la distancia, que indica que da igual estar más lejos si se dispone de cobertura y el trayecto es más rápido (Rietveld y Vickerman, 2004), cobra importancia. Por el contrario, los sectores no servidos por 
infraestructuras sufren de marginación espacial. Nos encontramos, pues, con un caso claro de anisotropía espacial, en el que las infraestructuras priorizan, y fomentan, el desarrollo de unos territorios en detrimento de otros.

El desplazamiento de los usos del suelo de menor valor añadido hacia la periferia, debido a la creciente especialización económica del centro, no es ajeno a la disposición infraestructural. Siguiendo el marco teórico de los factores de localización, la proximidad a infraestructuras metropolitanas de primer nivel, como carreteras locales, ha condicionado la ubicación de centros comerciales o de industrias de alto valor añadido, entre otros. Por otro lado, la proximidad a infraestructuras de conexión regional, como autopistas o líneas de ferrocarril, ha atraído centros logísticos, servicios aeroportuarios, puertos secos, etc. En términos residenciales, los sectores de renta alta se han ubicado en terrenos con mejores niveles de conectividad, mientras que los de renta baja han quedado relegados a las zonas peor conectadas. En cualquier caso, muchos de estos ciudadanos continúan manteniendo relaciones con la ciudad central, y estas se vehiculan a través de estas redes infraestructurales.

Por todos estos motivos resulta conveniente debatir sobre las políticas de movilidad aplicadas a las grandes ciudades para mejorar la sostenibilidad global del sistema. La implantación de peajes urbanos directos, o encubiertos (por ejemplo, el pago por estacionar), las restricciones de acceso al vehículo privado o los cambios en el modelo viario contribuyen a reducir el tráfico y a mejorar la calidad del aire en la ciudad central. El problema es que, sin una fuerte inversión en infraestructuras de transporte público, la periferia pierde accesibilidad gradualmente. Se conforma un territorio todavía más desigual, donde los desplazados cautivos del vehículo privado son penalizados doblemente. Debido a las nuevas políticas implementadas, el tiempo y el coste dedicado a la movilidad aumentan, y la calidad de vida de los ciudadanos de la periferia se reduce.

Los desplazados sufren las consecuencias de un planeamiento urbano que no ha sabido entender los procesos migratorios y que, durante mucho tiempo, ha mantenido el enfoque en la escala local, sin entender la ciudad como un espacio de relaciones, y no solamente como espacio físico. Hace tiempo que las ciudades han pasado a ser entidades territoriales y, por lo tanto, las políticas urbanas no se pueden limitar al contexto de la ciudad como centro, sino que deben pensarse sobre el conjunto metropolitano.

\section{La necesidad de un cambio de modelo: hacia un crecimiento urbano más sostenible}

ONU Hábitat define el índice de prosperidad de la ciudad sobre la base de cinco indicadores:

1) productividad;

2) desarrollo de las infraestructuras;

3) calidad de vida;

4) equidad e inclusión social, y

5) sostenibilidad medioambiental.

Optar por un modelo de crecimiento más sostenible nos obliga a repensar el proceso de urbanización imperante en las últimas décadas, especialmente en relación con la ocupación del territorio y el despliegue infraestructural.

La primera recomendación pasa por entender las ciudades de manera diferente. Hay que dar un salto de escala, del municipio al área metropolitana, incluyendo los usos del suelo, pero también sus relaciones. Las medidas implementadas sobre un ámbito concreto tienen ramificaciones y acaban repercutiendo en el resto. Solo si entendemos que la ciudad no acaba donde finaliza su límite físico, sino que esta no existe sin su área de influencia y la dependencia de otras ciudades, seremos capaces de planificar los nuevos crecimientos de manera sostenible. Sin embargo, si continuamos pensando de manera local, el resultado será un territorio cada vez más fragmentado y desigual.

Hay que repensar morfológicamente la ciudad. Las metrópolis no se pueden entender como elementos continuos y monocéntricos, como exponían los modelos teóricos de hace unos cuantos años, pero sí que continúa vigente la necesidad de buscar el equilibrio entre actividades económicas y zonas residenciales. Reducir las distancias entre los diferentes polos económicos y sociales es la única manera de minimizar el coste ambiental de la ciudad construida. El concepto teórico de la ciudad de los 15 minutos, es decir, que todo ciudadano pueda realizar todas las actividades diarias desplazándose un máximo de 15 minutos, va en esta línea (Mardones et al., 2020). En este sentido, la planifi- 
cación de nuevos sectores urbanos ha de tener en cuenta la mixtura de usos. Garantizar sectores bien conectados, asegurar un aprovisionamiento logístico sostenible, reducir la marginación espacial, hacer los barrios más habitables, apostar por una proporción más grande de verde dentro de la ciudad, incrementar la autosuficiencia energética y de recursos, o aproximar los sectores de producción y agrícolas serán también necesarios para el logro de unos umbrales de desarrollo más sostenible.

El papel de las infraestructuras es también clave para lograr un desarrollo más sostenible. La planificación conjunta de infraestructuras y usos del suelo debe articular un territorio mejor integrado, con menos vacíos de accesibilidad y con una conexión más eficiente entre todos los nodos. Esto requiere que las diferentes administraciones se coordinen y abandonen ideas como la planificación a escala municipal o el planeamiento sectorial. En el ámbito local, hay que resolver el paso de las infraestructuras por la ciudad, asegurar la integración con la trama urbana y evitar la creación de vacíos de cobertura que lleven a la degradación del espacio urbano. En este sentido, el uso de herramientas como el desarrollo orientado al transporte público (DOT) nos debe permitir planificar las infraestructuras garantizando la sostenibilidad de los nuevos sectores.

En términos territoriales, hay que poner freno a los procesos de urbanización que llevan a la conversión de suelo no urbanizable en suelo urbano. Solo de este modo conseguiremos preservar el medio natural y los corredores de conectividad ecológica. El desarrollo de nuevos sectores se debería restringir a casos excepcionales y debidamente justificados, preferiblemente en áreas bien conectadas por las infraestructuras de transporte, y asegurando todos los estándares de calidad, sostenibilidad y equidad social.

En la ciudad consolidada, se pueden aplicar soluciones que ayuden a mejorar la calidad de vida y la sostenibilidad del espacio construido. Promover la rehabilitación de los espacios centrales abandonados ayudará a aumentar la mixtura de usos y a crear un entorno urbano más agradable y eficiente. En la renovación de estos espacios, se puede incentivar la vivienda social, la habilitación de áreas verdes y huertos urbanos, y la concesión de más espacio público para bicicletas y peatones. En el ámbito de las infraestructuras de servicios públicos, por ejemplo, se pueden introducir sistemas urbanos de drenaje sostenible (SUDS), promover cubiertas verdes en los edificios, implantar puntos de recogida selectiva de residuos o instalar placas solares en elementos del mobiliario urbano. Estas medidas se deben entender dentro de las limitaciones de los instrumentos urbanísticos. Rehabilitar y reurbanizar un barrio requiere grandes inversiones económicas. En este sentido, desde el urbanismo táctico, se proponen actuaciones temporales, de bajo coste, pero que permiten incentivar un cambio de hábitos en la ciudadanía (Mould, 2014). Se trata de un proceso que permite experimentar diferentes soluciones sin hipotecar el espacio urbano, además de observar los cambios introducidos y las dinámicas resultantes para, después, proyectar de la manera más adecuada. Este tipo de herramientas entienden la ciudad como un laboratorio a cielo abierto y evitan el dispendio de recursos públicos en soluciones que pueden no tener los efectos esperados.

La apropiación del espacio urbano degradado mediante programas de participación ciudadana también es una herramienta útil y eficaz para renovar la ciudad utilizando pocos recursos. El uso de materiales reciclados, o la reutilización de materiales sobrantes de obras ya ejecutadas (como galerías de hormigón, palets, etc.), contribuyen a reducir el impacto de los procesos de renovación y a la vez contener el gasto público. Por otro lado, la creación de talleres locales para la construcción de mobiliario urbano o para el diseño colaborativo del espacio ayudan a crear espacios más arraigados entre los residentes, que después de participar en su transformación se sienten corresponsables.

La introducción de nuevas tecnologias también puede contribuir a mejorar la sostenibilidad de la ciudad, por ejemplo, por las posibilidades de trabajar remotamente que confieren y con ello permitir la reducción del número de viajes necesarios. Las mejoras tecnológicas se pueden aplicar también para mejorar la gestión de las redes o para promover el acceso popular a las nuevas tecnologías TIC (por ejemplo, instalación de puntos de wifi en diferentes puntos de la ciudad). O, incluso, pueden servir para fomentar las prácticas de compartición. Por ejemplo, nuevas políticas de economía colaborativa que incentiven las comunidades de vecinos con recursos compartidos pueden contribuir a reducir el gasto energético, minimizar los residuos y crear un entorno social más cohesionado. Además, el uso compartido de viviendas puede ayudar a contener la demanda y permitir reducir las migraciones hacia la periferia causadas por el elevado coste de la vivienda. 


\section{Referencias bibliográficas}

ALVAREZ-PALAU, Eduard; MARTÍ, Jordi; SOLANAS, Jorge (2019). «Urban Growth and Long-Term Transformations in Spanish Cities Since the Mid-Nineteenth Century: A Methodology to Determine Changes in Urban Density». En: Sustainability, vol. 11, núm. 6948 [en línea]. DOl: https://doi.org/10.3390/su11246948

HERCE, Manuel (2013). El negocio del territorio: evolución y perspectivas de la ciudad moderna, pág. 388. Madrid: Alianza.

HERCE, Manuel; MIRÓ, Joan (2002). El soporte infraestructural de la Ciudad, pág. 186. Barcelona: Universidad Politécnica de Cataluña.

KOPNINA, Helen (2016). «The victims of unsustainability: a challenge to sustainable development goals». En: International Journal of Sustainable Development \& World Ecology, vol. 23, núm. 2, págs. 113-121 [en línea]. DOI: https:// doi.org/10.1080/13504509.2015.1111269

MARDONES, Nuño; LUQUE, José; ASEGUINOLAZA, Izaskun (2020). «La ciudad del cuarto de hora, ¿una solución sostenible para la ciudad postCOVID-19?». En: Ciudad y Territorio, núm. 205, págs. 653-664.

MOULD, Oli (2014). «Tactical urbanism: The new vernacular of the creative city». En: Geography compass, vol. 8, núm. 8, págs. 529-539 [en línea]. DOI: https://doi.org/10.1111/gec3.12146

RIETVELD, Piet; VICKERMAN, Roger (2004). «Transport in regional science: The death of distance is premature». En: Papers in Regional Science, vol. 83, págs. 229-248 [en línea]. DOI: https://doi.org/10.1007/978-3-662-07223-3_10

NACIONES UNIDAS (ONU), Comisión de Población y Desarrollo (2018). Ciudades sostenibles, movilidad humana y migración internacional. Informe del Secretario General de la ONU (24 pág.).

NACIONES UNIDAS (ONU), Departamento de Asuntos Económicos y Sociales (2019). World Urbanization Prospects: The 2018 Revision (ST/ESA/SER.A/420). Nueva York: Naciones Unida

Cita recomendada: HERNÁNDEZ ASENSI, Mireia; ALVAREZ-PALAU, Eduard J. Urbanización, ocupación del suelo e infraestructuras de soporte. Oikonomics [en línea]. Noviembre 2021, n. 16. ISSN: 2339-9546. DOI: https://doi.org/10.7238/o.n16.2113 


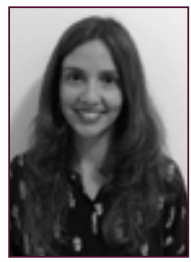

\section{Mireia Hernández Asensi}

mhernandezas@uoc.edu

Directora de proyectos en PCE Engenharia. Profesora colaboradora en el Máster de Gestión de la Ciudad y Urbanismo (UOC)

Ingeniera de Caminos, Canales y Puertos con especialidad en urbanismo y transportes, y tiene los posgrados de Gestión Privada de Infraestructuras y Elementos de Planificación Urbana por la Universidad Politécnica de Cataluña (UPC). Profesora colaboradora del máster Ciudad y Urbanismo $(\cup O C)$. Tiene más de diez años de experiencia en planificación y diseño de infraestructuras del transporte, planificación urbana, drenaje y urbanización. Ha dirigido proyectos en Europa y en América Latina para empresas privadas e instituciones públicas. Trabaja como directora de proyectos en PCE Projetos e Consultorias de Engenharia Ltda (Río de Janeiro). Anteriormente, trabajó como directora de proyectos e ingeniera proyectista en Barcelona, São Paulo y Brasilia.

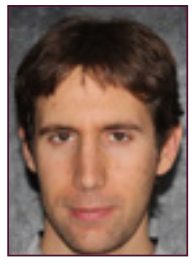

\section{Fduard J. Alvarez-Palau}

ealvarezp@uoc.edu

Profesor de los Estudios de Economia y Empresa (UOC)

Doctor en Ingeniería e Infraestructuras de Transporte (UPC), ingeniero de Caminos, Canales y Puertos (UPC) y máster de Dirección de Organizaciones (UOC). Profesor lector de los Estudios de Economía y Empresa de la UOC y miembro del grupo de investigación Sustainability and Management (SUMA). Anteriormente, ha sido investigador posdoctoral en la Universidad de Cambridge, becario de la Comisión Europea (DG MOVE), profesor asociado de la UPC y consultor externo de la UOC. Tiene también experiencia en la dirección de proyectos de ingeniería civil, planificación urbanística y de transportes en la empresa privada.

Los textos publicados en esta revista están sujetos -si no se indica lo contrario- a una licencia de Reconocimiento 4.0 Internacional de Creative Commons. Puede copiarlos, distribuirlos, comunicarlos públicamente, hacer obras derivadas siempre que reconozca los créditos de las obras (autoría, nombre de la revista, institución editora) de la manera especificada por los autores o por la revista. La licencia completa se puede consultar en https://creativecommons.org/licenses/by/4.0/deed.es_ES.

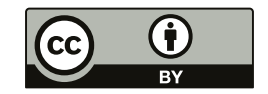

